\title{
Synthesis and Characterization of LiZnP and LiZnAs Semiconductor Material
}

Benjamin W. Montag ${ }^{1}$, Michael A. Reichenberger ${ }^{1}$, Kevin R. Arpin ${ }^{1}$, Madhana Sunder ${ }^{2}$, Kyle A. Nelson ${ }^{1}$, Philip B. Ugorowski ${ }^{1}$, Douglas S. McGregor ${ }^{1}$

${ }^{1}$ Semiconductor Materials and Radiological Technologies (S.M.A.R.T) Laboratory, Kansas State University, Manhattan, KS 66506, U.S.A.

${ }^{2}$ Bruker AXS Inc, 5465 E. Cheryl Parkway, Madison, WI 53711, U.S.A.

\section{ABSTRACT}

Research for a reliable solid-state semiconductor neutron detector continues because such a device has not been developed, and would have greater efficiency, than present-day gas-filled ${ }^{3} \mathrm{He}$ and ${ }^{10} \mathrm{BF}_{3}$ neutron detectors. Further, a semiconductor neutron detector would be more compact and rugged than most gas-filled or scintillator neutron detectors. The ${ }^{6} \mathrm{Li}(\mathrm{n}, \mathrm{t})^{4} \mathrm{He}$ reaction yields a total $\mathrm{Q}$ value of $4.78 \mathrm{MeV}$, a larger yield than the ${ }^{10} \mathrm{~B}(\mathrm{n}, \alpha)^{7} \mathrm{Li}$, and is easily identified above background radiation interactions. Hence, devices composed of either natural $\mathrm{Li}$ (naturally $7.5 \%{ }^{6} \mathrm{Li}$ ) or enriched ${ }^{6} \mathrm{Li}$ (approximately 95\% ${ }^{6} \mathrm{Li}$ ) may provide a semiconductor material for compact high-efficiency neutron detectors. A sub-branch of the III-V semiconductors, the filled tetrahedral compounds, known as Nowotny-Juza compounds $\left(\mathrm{A}^{\mathrm{I}} \mathrm{B}^{\mathrm{II}} \mathrm{C}^{\mathrm{V}}\right)$, are desirable for their cubic crystal structure and semiconducting electrical properties. These compounds were originally studied for photonic applications. In the present work, Equimolar portions of $\mathrm{Li}, \mathrm{Zn}$, and $\mathrm{P}$ or As were sealed under vacuum $\left(10^{-6}\right.$ Torr $)$ in quartz ampoules with a boron nitride lining, and loaded into a compounding furnace. The ampoule was heated to $200^{\circ} \mathrm{C}$ to form the $\mathrm{Li}-\mathrm{Zn}$ alloy, subsequently heated to $560^{\circ} \mathrm{C}$ to form the ternary compound, LiZnP or LiZnAs, and finally annealed to promote crystallization. The chemical composition of the synthesized starting material was confirmed at Galbraith Laboratories, Inc. by Inductively Coupled Plasma Optical Emission Spectroscopy (ICP-OES), which showed the compounds can be reacted in equal ratios, 1-1-1, to form ternary compounds. Recent additions to the procedure have produced higher yields, and greater synthesis reliability. Synthesized powders were also characterized by $\mathrm{x}$-ray diffraction, where lattice constants of $5.751 \pm .001 \AA$ and $5.939 \pm .002 \AA$ for LiZnP and LiZnAs, respectively, were determined.

\section{KEYWORDS}

B1. Lithium Compounds; A1. Radiation; B2. Semiconducting ternary compounds; A1. X-ray diffraction; A1. Crystal structure

\section{INTRODUCTION}

The filled tetrahedral compound class $\mathrm{A}^{\mathrm{I}} \mathrm{B}^{\mathrm{II}} \mathrm{C}^{\mathrm{V}}$ materials consist of the III-V-like compounds with Li interstitials, also referred to as Nowotny-Juza compounds. Nowotny-Juza compounds were originally, and still today, studied for photonic applications [1-3]. The zincblende crystal structure is arranged where the group II atom is located at $\tau_{1}=(0,0,0) a$ where $a$ is the lattice constant, and the group $\mathrm{V}$ atom is located at $\tau_{2}=(1 / 4,1 / 4,1 / 4) a$. The 
spacious cubic structure allows for lithium atoms to fill its interstitial site at $\tau_{3}=(1 / 2,1 / 2,1$ / 2) $a$, as shown in Fig. 1 [4-6]. The filling of these interstitial sites allows for a Li-loaded semiconducting material. Useful electrical properties have been reported by optical means, where LiZnP and LiZnAs have a direct bandgap of $2.1 \mathrm{eV}$ and $1.51 \mathrm{eV}$, respectively [4, 6].

Nowotny-Juza materials are interesting for solid-state neutron detectors because the devices would be rugged, operate at ambient temperature, have high thermal neutron detection efficiency, and adequate gamma-ray rejection. Materials containing, ${ }^{6} \mathrm{Li},{ }^{10} \mathrm{~B},{ }^{113} \mathrm{Cd},{ }^{157} \mathrm{Gd}$, and ${ }^{199} \mathrm{Hg}$ have been considered for solid-state neutron detectors [7-18]. The ${ }^{10} \mathrm{~B}(\mathrm{n}, \alpha){ }^{7} \mathrm{Li}$ reaction is desirable for the ${ }^{10} \mathrm{~B}$ microscopic thermal neutron absorption cross section of 3839 barns, but boron-based compounds, such as BP, BN, and BAs, have shown limited success and thus far do not appear promising. These boron based materials typically fail due to crystal growth, purity, and materials preparation problems [15-19]. A high purity semiconductor is extremely important to reduce charge trapping, capacitance, and other charge transport related problems that greatly degrade the device performance. Additionally, thin-film coated boron devices suffer low neutron detection efficiency due to their geometry, where only one reaction product is absorbed in the semiconducting material. Consequently, the resultant signals may be small and difficult to distinguish from background and gamma-ray induced events [20]. Additionally, depending on the range of the reaction products in the boron material, film thickness is restricted, due to energy self-absorption, resulting in a maximum intrinsic detection efficiency of approximately $4.5 \%$ [20]. Solid-state detectors containing ${ }^{113} \mathrm{Cd}$ and ${ }^{199} \mathrm{Hg}$ devices also have limited detection efficiency due to the low absorption probability of the prompt gamma-rays that result from the reactions ${ }^{113} \mathrm{Cd}(\mathrm{n}, \gamma){ }^{114} \mathrm{Cd}$ and ${ }^{199} \mathrm{Hg}(\mathrm{n}, \gamma){ }^{200} \mathrm{Hg}$ [7-10]. The reaction ${ }^{157} \mathrm{Gd}(\mathrm{n}, \gamma){ }^{158} \mathrm{Gd}$ is desirable for the large ${ }^{157} \mathrm{Gd}$ thermal neutron capture cross section of 259,000 barns [21]. The reaction results in low-energy prompt gamma rays and conversion electrons below $220 \mathrm{keV}$, which are difficult to discern from background radiations [20]. Finally, ${ }^{6} \mathrm{Li}$ has not been explored to the same extent as other thermal neutron absorbers, and has an intrinsic thermal neutron absorption cross section of 940 barns. The reaction produces a total Q-value of $4.78 \mathrm{MeV}$, shown in the following reaction,

$$
{ }^{6} \mathrm{Li}+{ }_{0}^{1} n \rightarrow{ }^{3} \mathrm{H}(2.73 \mathrm{MeV})+\alpha(2.05 \mathrm{MeV}) .
$$

Many Nowotny-Juza compounds have been synthesized in the past, however, difficulties in synthesis, purification, crystal growth, and handling, unfortunately limit the available physical and electrical property data. These materials have traditionally been reacted in a one, or two-step process to synthesize the ternary material [1-6, 22-25]. Ternary (LiZnP, LiZnAs) and binary materials $\left(\mathrm{Li}_{3} \mathrm{As}, \mathrm{Li}_{3} \mathrm{P}\right)$ are not commercially available, and therefore require in-house techniques to acquire desired materials. Given the reactive nature of $\mathrm{Li}$, careful consideration of material synthesis and crystal growth must be made. Additionally, observed melting temperatures of these ternary compounds exceed that of conventional Bridgman/Stockbarger furnaces $\left(>1200^{\circ} \mathrm{C}\right)$. Thus, clever synthesis and bulk growth techniques are required for single crystal growth. In following study, a three step reaction process was used, which reduced the nature of the exothermic reaction that occurs, and as result, greatly increased the ampoule yield from cracking or exploding during the reaction process.

\section{EXPERIMENTAL DETAILS}


The following sections detail the synthesis process and crystallization of LiZnAs and LiZnP. Through rigorous experimentation, the following synthesis procedure produced the most consistent $\mathrm{A}^{\mathrm{I}} \mathrm{B}^{\mathrm{II}} \mathrm{C}^{\mathrm{V}}$ material and ICP-OES and X-ray diffraction studies confirmed material content and crystal structure.

\section{I. $\quad$ Synthesis Process}

LiZnP and LiZnAs multicrystalline samples were synthesized from elemental material, 99.995\% zinc powder (Sigma-Aldrich), 99.999\% phosphorus chunk (Alpha-Aesar), 99.999\% arsenic chunk (Alpha-Aesar), and 99.9\% lithium rod (ESPI metals). The elemental materials were loaded into pyrolitic boron nitride $(\mathrm{pBN})$, tantalum, or graphite crucibles under an argon atmosphere. Phosphorus pieces were dried in an argon environment over a hot plate in a tantalum crucible, and then crushed into a fine powder with a clean mortar and pestle. Lithium pieces approximately $2 \mathrm{~mm}$ thick were cut from the rod, and the sides where oxidation had progressed were also removed. The remaining large pieces were sectioned into $1-3 \mathrm{~mm}^{3}$ samples, and cleaned of residual oil in anhydrous hexane. Crucibles were cleaned of oil and other contaminants by ultra-sonic vibration in hexane, trichloroethylene, acetone, and isopropanol separately. Boron nitride porosity is minimal compared to what is observed with graphite materials, where the porosity is in the range of $9-12 \%$ depending on the material grade [26]. Lithium becomes very reactive once molten, and can react with graphite to make lithium carbides. Graphite has historically been used in the synthesis process [24], although a more repeatable process was found by using a pyrolitic boron nitride crucible, or sealed tantalum under argon. Lithium (40\% atom weight) and zinc (60\% atom weight) was weighed and added to the crucible as described by the Li-Zn phase diagram [27]. An equimolar portion of phosphorus or arsenic was added to the $\mathrm{Li}, \mathrm{Zn}$ mixture. An excess of 3-5\% lithium was included to account for evaporative loses during the reaction process. The crucible, and a quartz plug, were loaded into a quartz ampoule and transferred to a vacuum station while remaining under argon using a vacuum valve attachment. The ampoule was evacuated to approximately $9.0 \times 10^{-6}$ Torr beginning with a roughing pump vacuum followed by an Alcatel ATP 80 turbo pump evacuation. Vapor pressure of phosphorus limits the evacuation pressure achievable. It is known that after heating to only $90{ }^{\circ} \mathrm{C}$, phosphorus vapor pressure rises to approximately $9.9 \times 10^{-6}$ Torr [28]. The ampoule was sealed using a hydrogen / oxygen torch.

The sealed ampoule was placed into a rotation-stage furnace system, where the ampoule was rotated at approximately 0.25 rotations per second at approximately a $45^{\circ}$ angle to promote mixing. The furnace was ramped at $1^{\circ} \mathrm{C}$ per minute to $200^{\circ} \mathrm{C}$ while the ampoule was rotated to form the alloy Li-Zn [27] and held for 24 hours. The rotation was stopped, and the furnace was ramped at $0.5^{\circ} \mathrm{C}$ per minute to $560^{\circ} \mathrm{C}$ to promote ternary reaction. The furnace was held for 48 hours, and then ramped to $720^{\circ} \mathrm{C}$ or $660^{\circ} \mathrm{C}$ for $\mathrm{LiZnP}$ or LiZnAs respectively to anneal and promote crystallization for another 48 hours. The furnace was then ramped to room temperature at $1^{\circ} \mathrm{C}$ per minute. The ampoule was removed from the furnace, and placed in an argon glove box where the material could be extracted and stored. Typical reactions yielded approximately $2.0 \mathrm{~g}$ of material. LiZnAs reactions were scaled up to yield up to approximately $6.0 \mathrm{~g}$, although LiZnP reactions had to remain in much smaller batches $(2.0 \mathrm{~g})$ due to problems with ampoule cracking observed with larger batches.

\section{Characterization}




\section{RESULTS}

The exothermic reaction that results from synthesizing Nowotny-Juza compounds stresses the quartz containment, often resulting in cracking and a loss of material. By producing the alloy, Li-Zn, before reaction, a much more stable reaction was achieved, resulting in a much higher harvesting yield, where cracked, or exploded ampoules were reduced. Shown in Fig. 3 are the ampoules after reactions for cases of cracking and non-cracking. Cracking usually forms at the bottom of the ampoule, and resulting in oxidized lithium and crucible material.

From a successful reaction, the resulting synthesized material can be seen in Fig. 4, which produced powder and chunks usually up to approximately $1.0 \mathrm{~mm}$ across, and in some cases, facets that were quite large, up to $7.5 \mathrm{~mm}$ in diameter. Three samples, one LiZnP and two LiZnAs, were sent to Galbraith Laboratories Inc. for inductively coupled plasma optical emission spectroscopy analysis (ICP-OES) to examine elemental concentration after synthesis to provide an indication of congruent reaction. The ICP-OES process was performed as described by the EPA, Method 6010b [31]. The ICP-OES results are shown in Table 1. The analysis column is the constituent element measured, where the resulting spectroscopy is presented as weight percent. Each weight percent was divided by the element molar mass to find the elemental molar mass concentration within the sample. Analysis of each constituent element is associated with a $10 \%$ relative standard error as stated by Galbraith Laboratories Inc.

Numerous powder x-ray diffraction phase identification scans were collected for LiZnP and LiZnAs powders on a Bruker AXS D8 ADVANCE with $\mathrm{Cu}$ radiation, using the Bragg Brentano geometry. Examples of the collected patterns can be seen for LiZnAs and LiZnP in Fig. 
6 and Fig. 7, respectively. Both patterns matched well with what was reported by Nowotny and Bacewicz cited in the International Center for Diffraction Data (ICDD) [2, 24]. Lattice constants were determined using TOPAS (Bruker AXS Inc). The Whole Powder Pattern decomposition approach was used. Refinement of the experimental phase identification data yielded lattice constants of $5.751 \pm 0.001 \AA$ and $5.939 \pm 0.002 \AA$ for LiZnP and LiZnAs, respectively.

Cleaved facet sample crystals were analyzed for evidence of crystallinity using a Bruker AXS D2 CRYSO energy dispersive x-ray diffractometer (EDXRD). The instrument scans the sample at multiple positions of $\square$ angles, where a family of planes can be identified through a series of calculations [32]. For a particular LiZnAs sample, the (111) family of planes were identified as seen in Fig. 8, where each spectrum is a measurement at a different position of phi across 360 degrees. A $d$ spacing of $1.755 \AA$ was determined from the location (energy) of these peaks, and the orientation and lattice constant of the sample. Characteristic x-rays are also collected by this method providing an indication of impurities in the sample. Characteristic $\mathrm{x}$ rays from calcium, copper and iron were observed from the LiZnAs sample.

The crystallinity of the (111) family of planes was examined by carrying out rocking curve measurements using a 4 bounce monochromator, with a line beam $12.0 \mathrm{~mm}$ tall by $9.5 \mathrm{~mm}$ width (slit size). The full width of half maximum of the (111) atomic plane was determined to be $0.097^{\circ}$ as seen in Fig 9. A rocking curve performed on a single crystal silicon standard (from Bruker AXS) yielded a FWHM of 0.004 degrees (14.4 arc seconds) for the Si (111) reflection, which indicates that the rocking curve FWHM broadening of the LiZnAs sample is primarily coming from the mosaicity of the sample. Finally, a phase identification scan was collected on the sample shown in Fig. 10. The (111) LiZnAs reflection is quite prominent at approximately $25.5^{\circ} 2 \theta$ and in addition there is indication of the (222) LiZnAs reflection at approximately $52^{\circ}$ 20. Samples were prepared under argon, and sealed using an X-ray transparent Mylar® film, in order to protect the samples from degradation during the measurement process.

\section{DISCUSSION \& CONCLUSIONS}

Nowotny-Juza compounds were synthesized into powder and large pieces, and ICP-OES confirmed the materials can be synthesized into a 1-1-1 ratio. As seen in Table 1, the total weight percent of each sample equaled approximately $100 \%$ within the $10 \%$ standard error that is associated with each elemental measurement. The sample with the most deviation from a total of 100\% was LiZnAs (1) with 78.23\%. Additionally, the elemental molar concentration also deviated most between constituent elements out of the three samples tested. These two deviations raise concerns that some synthesis reactions may result in incongruent reaction, and contain more unreacted elemental material, or binary materials, which indicates that not each ternary reaction produces a consistent 1-1-1 stoichiometric ratio of constituent elements.

Powder XRD phase identification scans also confirmed the formation of cubic $\mathrm{A}^{\mathrm{I}} \mathrm{B}^{\mathrm{II}} \mathrm{C}^{\mathrm{V}}$ materials for both LiZnAs and LiZnP, where the correlation between the measured and archival peaks clearly show that both materials were formed. The reflections shown in Fig. 6 and 7 matched well with theoretical pattern calculated using PowderCell 2.4, as shown in Fig. 5, and also matched with the experimental patterns included in the ICDD powder diffraction data base $[2,24]$. Not all peaks can be identified in Fig. 6 and 7, which may indicate contamination in the synthesized powders. One indication of contamination in Fig. 6 is observed where peaks match with profiles from lithium oxide, shown in the vertical dashed lines. Other impurity peaks are 
consistent with profiles from lithium carbonate, lithium arsenide, lithium phosphide, and lithium hydroxide. This contamination likely occurred during the handling and measurement process, given the material is hygroscopic In addition, the lithium rod that was used in the synthesis process provided by ESPI metals had a $99.9 \%$ purity. From the trace element analysis, $99.9 \%$ lithium contains traces of elements including calcium $(100 \mathrm{pm})$, iron $(10 \mathrm{ppm})$, and copper $(<30$ ppm) [33] which were observed in the EDXRD spectra shown in Fig 8. Additionally, with the current synthesis method, it is suspected that unreacted elemental material, possible incomplete binary materials, or other undesired compounds from possible reaction with the crucible lining material, may be present in the reaction products; therefore purification of these synthesized materials must be performed for a material grade needed for semiconductor devices.

Large single crystal facets grown in the synthesis process did exhibit in- plane crystalline ordering. A rocking curve collected on a LiZnAs sample of (111) planes is shown in Fig. 9. However, the rocking curve peak profile is not perfectly Gaussian and is indicative of other domains/facets in the sample. Evidence of other domains was also seen from the phase identification scan shown in Fig. 10. The most prominent peaks, at $25.5^{\circ}$ and $52^{\circ}$, show presence of the (111) and (222) LiZnAs reflections as expected. However, extremely low intensity peaks are present at approximately $38.5^{\circ}$ and $44.7^{\circ}$, which indicate presence of other domains in the particular sample.

The desired ternary Nowotny-Juza materials were synthesized from elemental constituents. The material exhibited the cubic crystal structure with atom placement in the desired locations and lattice constants consistent with calculations and experimental data. However, material purity is not ideal as indicated from the ICP-OES analysis and the XRD phase identification scans. Crystals with in-plane ordering can be found in amongst the synthesized powders and samples. These samples exhibited crystallinity and a reasonable rocking curve FWHM value for initial growths. Characterization of the synthesized powders is necessary for the development of a neutron detector-grade material.

\section{ACKNOWLEGEMENTS}

The authors express gratitude to Bruker AXS for allowing the use of their XRD instrumentation. The authors also express gratitude to John Desper, and the Kansas State University Chemistry department for the use of their Bruker D8 ADVANCE X-ray diffractometer. Work funded in part by the Advanced Materials program DOE - NNSA, grant \# DE-FG52-08NA28766.

\section{REFERENCES}

[1] R. Juza, K. Langer, and K. Von Benda, “Ternary Nitrides, Phosphides, and Arsenides of Lithium," Angewandte Chemie International Edition in English, vol. 7, no. 5, pp. 360370, 1968.

[2] H. Nowotny, and K. Bachmayer, "Die Verbindungen LiMgP, LiZnP und LiZnAs," Monatshefte für Chemie und verwandte Teile anderer Wissenschaften, vol. 81, no. 4, pp. 488-496, 1950. 
[3] D. Kieven, A. Grimm, A. Beleanu et al., "Preparation and properties of radio-frequencysputtered half-Heusler films for use in solar cells," Thin Solid Films, vol. 519, no. 6, pp. 1866-1871, 2011.

[4] K. Kuriyama, T. Kato, and K. Kawada, "Optical band gap of the filled tetrahedral semiconductor LiZnAs," Phys. Rev. B: Condens. Matter, vol. 49, no. 16, pp. 11452-5, 1994.

[5] K. Kuriyama, T. Katoh, and N. Mineo, "Crystal growth and characterization of the filled tetrahedral semiconductor lithium zinc phosphide (LiZnP)," J. Cryst. Growth, vol. 108, no. 1-2, pp. 37-40, 1991.

[6] K. Kuriyama, and T. Katoh, "Optical band gap of the filled tetrahedral semiconductor lithium zinc phosphide (LiZnP)," Phys. Rev. B: Condens. Matter, vol. 37, no. 12, pp. 7140-2, 1988.

[7] Z. W. Bell, K. R. Pohl, and L. Van Den Berg, "Neutron detection with mercuric iodide," IEEE Trans. Nuc. Sci., vol. 51, no. 3, pp. 1163-1165, 2004.

[8] A. G. Vradii, M. I. Krapivin, L. V. Maslova et al., "Possibilities of recording thermal neutrons with cadmium telluride detectors," Soviet Atomic Energy, vol. 42, no. 1, pp. 6466, 1977.

[9] A. G. Beyerle, and K. L. Hull, "Neutron detection with mercuric iodide detectors," Nucl. Inst. and Meth. A, vol. 256, no. 2, pp. 377-380, 1987.

[10] D. S. McGregor, J. T. Lindsay, and R. W. Olsen, "Thermal neutron detection with cadmium $_{1-\mathrm{x}}$ zinc $_{\mathrm{x}}$ telluride semiconductor detectors," Nucl. Inst. and Meth. A, vol. 381, no. 2, pp. 498-501, 1996.

[11] Y. Kumashiro, K. Kudo, K. Matsumoto et al., "Thermal neutron irradiation experiments on ${ }^{10} \mathrm{BP}$ single-crystal wafers," Journal of the Less Common Metals, vol. 143, no. 1-2, pp. 71-75, 1988.

[12] W. C. McGinnis, "Film Implementation of a Neutron Detector (FIND): Proof of Concept Device," Technical Report 1921, 2003.

[13] J. C. Lund, F. Olschner, F. Ahmed et al., "Boron Phosphide on Silicon for Radiation Detectors," MRS Online Proceedings Library, vol. 162, pp. 601-604, 1989.

[14] F. P. Doty, Boron Nitride Solid State Detector, US-6, 504, April 27, 2004, US-6,727,504, April 27, 2004.

[15] A. N. Caruso, P. A. Dowben, S. Balkir et al., "The all boron carbide diode neutron detector: Comparison with theory," Materials Science and Engineering: B, vol. 135, no. 2, pp. 129-133, 2006.

[16] B. W. Robertson, S. Adenwalla, A. Harken et al., "A class of boron-rich solid-state neutron detectors," Applied Physics Letters, vol. 80, no. 19, pp. 3644-3646, 2002.

[17] P. Groot, J. H. F. Grondel, and P. J. v. d. Put, "Chemical Vapour Deposition of Boron Phosphides Using Bromide Etchants," Solid State Ionics, vol. 16, pp. 95-98, 1985.

[18] A. N. Caruso, R. B. Billa, S. Balaz et al., "The Heteroisomeric Diode," J. Phys. Condens. Matter, vol. 16, pp. L139-L146, 2004.

[19] D. S. McGregor, T. C. Unruh, and W. J. McNeil, "Thermal neutron detection with pyrolytic boron nitride," Nuclear Instruments and Methods in Physics Research Section A: Accelerators, Spectrometers, Detectors and Associated Equipment, vol. 591, no. 3, pp. 530-533, 2008.

[20] D. S. McGregor, M. D. Hammig, Y. H. Yang et al., "Design considerations for thin film coated semiconductor thermal neutron detectors-I: basics regarding alpha particle 
emitting neutron reactive films," Nucl. Inst. and Meth. A, vol. 500, no. 1-3, pp. 272-308, 2003.

[21] NIST, "Neutron Scattering Lengths and cross sections," http://www.ncnr.nist.gov/resources/n-lengths/elements/gd.html, Feb 2014.

[22] K. Kuriyama, T. Kato, T. Kato et al., "Growth and photoluminescence properties of the filled tetrahedral semiconductor LiZnAs," J. Cryst. Growth, vol. 166, no. 1-4, pp. 631635, 1996.

[23] K. Kuriyama, and F. Nakamura, "Electrical transport properties and crystal structure of lithium zinc arsenide (LiZnAs)," Phys. Rev. B: Condens. Matter, vol. 36, no. 8, pp. 443941, 1987.

[24] R. Bacewicz, and T. F. Ciszek, "Preparation and characterization of some $\mathrm{A}^{\mathrm{I}} \mathrm{B}^{\mathrm{II}} \mathrm{C}^{\mathrm{V}}$ type semiconductors," Appl. Phys. Lett., vol. 52, no. 14, pp. 1150-1, 1988.

[25] R. Bacewicz, and T. F. Ciszek, "Crystal growth and characterization of some novel $\mathrm{A}^{\mathrm{I}} \mathrm{B}^{\mathrm{II}} \mathrm{C}^{\mathrm{V}}$ semiconductors," Acta Phys. Pol., A, vol. A77, no. 2-3, pp. 379-81, 1990.

[26] Mersen, "SPECIALITY GRAPHITE MATERIALS - FOR CONTINUOUS CASTING," http://www.mersen.com/uploads/tx_mersen/6-continuous-casting-mersen_06.pdf, Feb 2014.

[27] A. D. Pelton, "The Li-Zn (Lithium-Zinc) System," J. Phase Equi., vol. 12, no. 1, pp. 4245, 1991.

[28] R. E. Honig, "Vapor Pressure Data for the More Common Elements," RCA Rev., vol. 18, no. 2, pp. 195-204, 1957.

[29] H. E. Swanson, "Standard X-ray diffraction powder patterns," Washington, D.C.: U.S Government Printing Office, 1969.

[30] W. Kraus, and G. Nolze, "POWDER CELL - a program for the representation and manipulation of crystal structures and calculation of the resulting X-ray powder patterns," J. Appl. Crys., vol. 29, no. 3, pp. 301-303, 1996.

[31] U.S. Environmental Protection Agency, "Method 6010B," Inductively Coupled Plasma Atomic Emission Spectrometry, 1996.

[32] Bruker AXS Inc, "brochure_D2_CRYSO.pdf," http://bruker.poznan.pl/images/stories/AXS/brochure_D2_CRYSO.pdf, 2009.

[33] ESPI Metals, "Lithium Rod Typical Analysis," http://www.espimetals.com/index.php/component/content/article/378-llithium-li/392llithium-li.
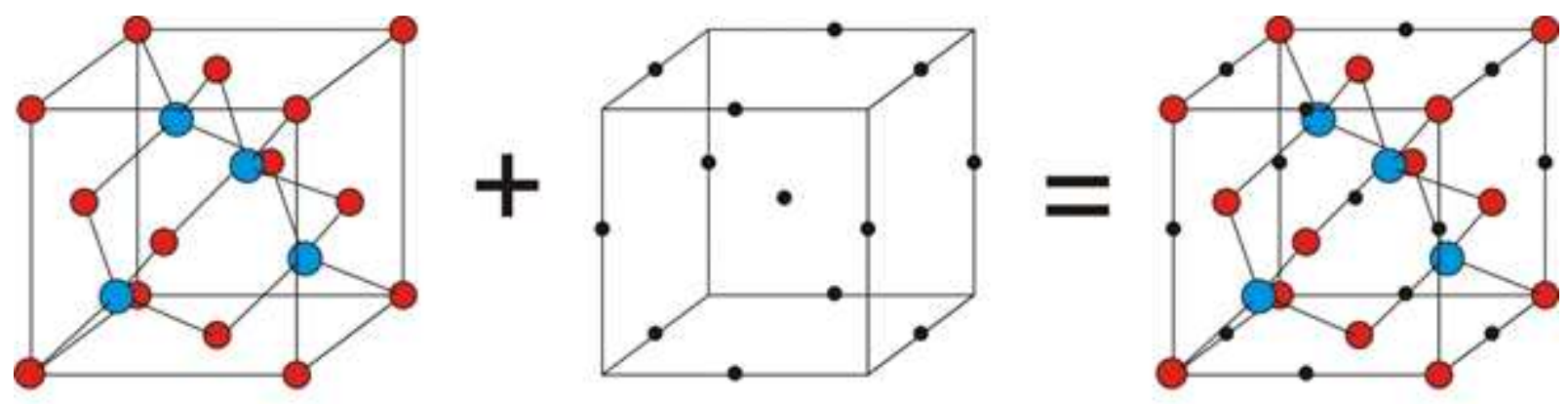
Fig. 3. Example of ampoule cracking (circled portion) (top), and example of a successful ternary material synthesis 364

Fig. 2. A LiZnAs facet prepared for XRD measurements in a sample holder between two Mylar® films.

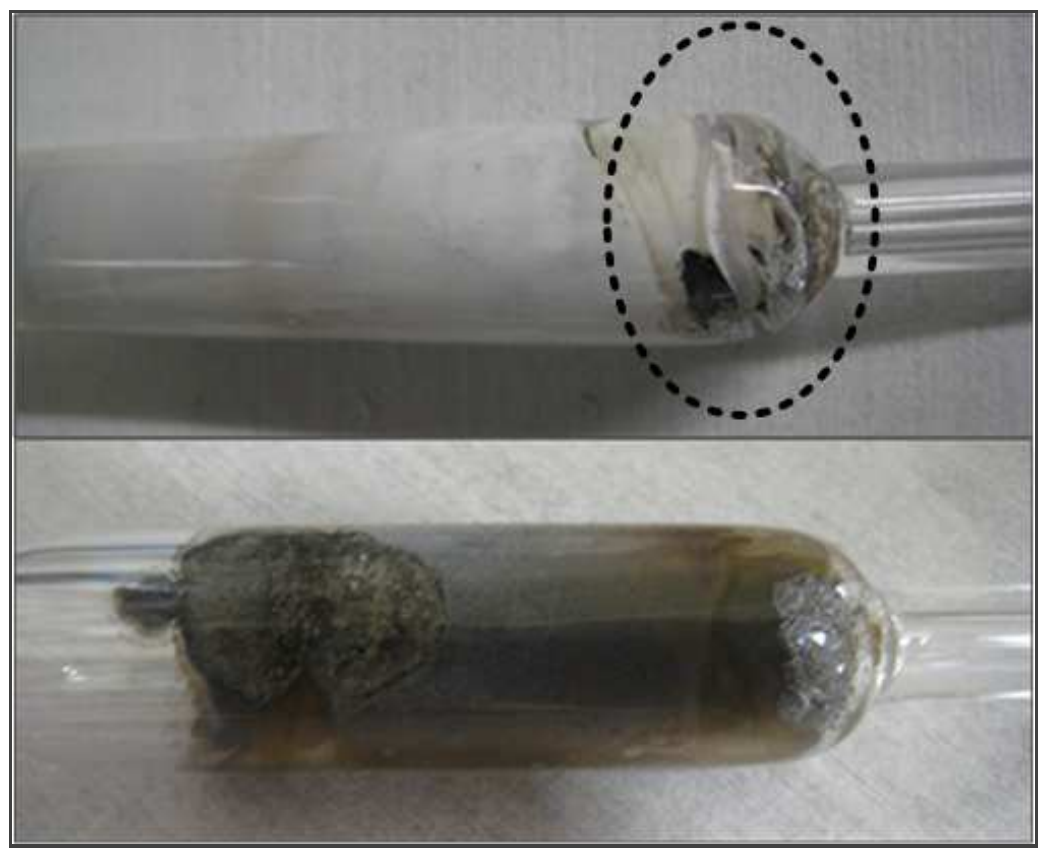
using a graphite crucible (bottom). 


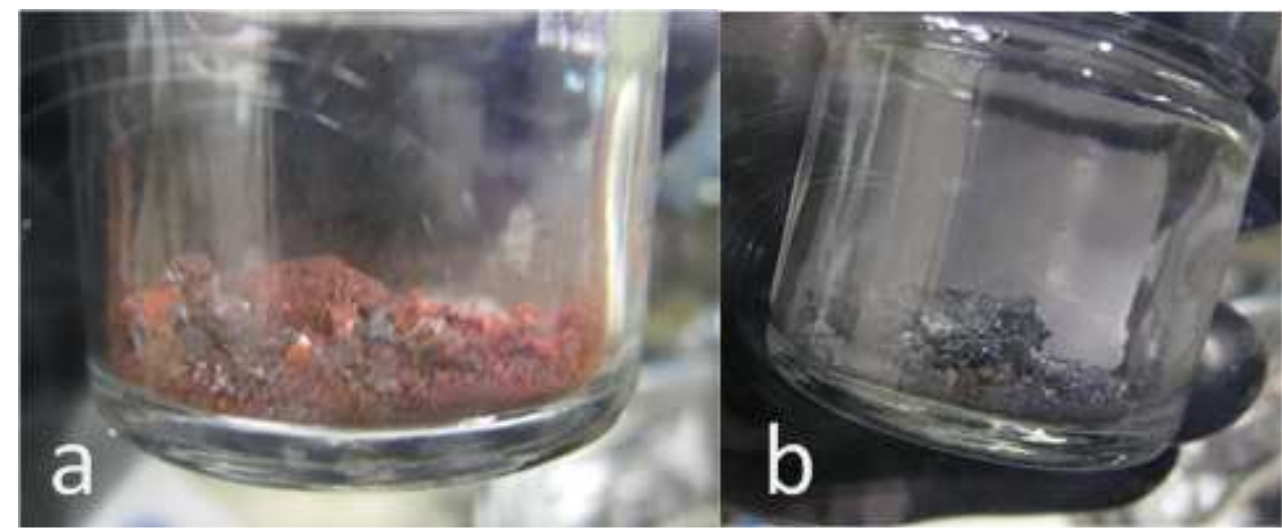

Fig. 4. Nowotny-Juza synthesized material. a. LiZnP red metallic power and chunks. b. LiZnAs black metallic powder and chunks.

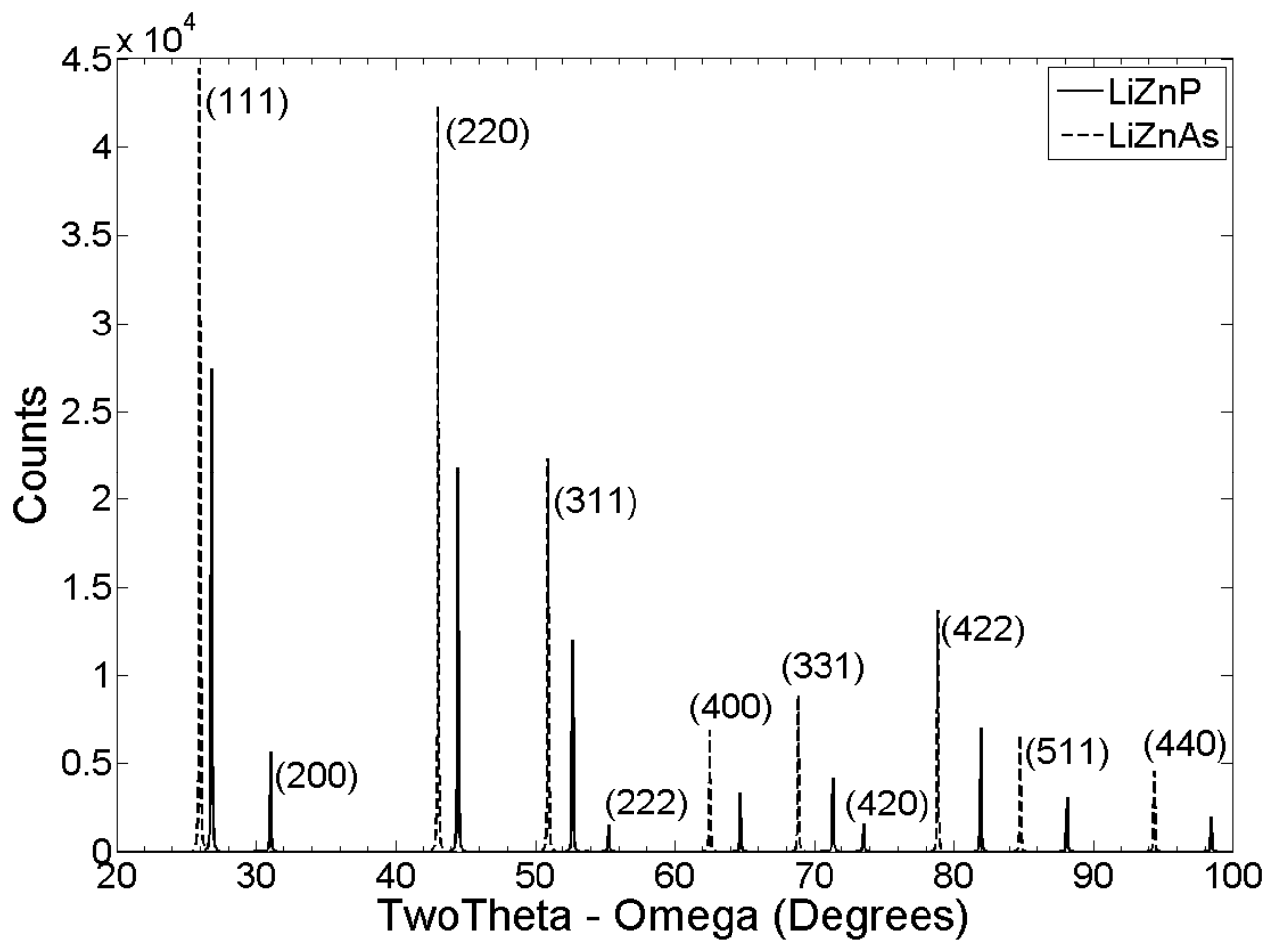

Fig. 5. The theoretical $\mathrm{Cu}$ x-ray phase identification of LiZnP (blue) and LiZnAs (dashed) powders calculated with PowderCell 2.4[30]. Lattice constants and space groups were used in the calculation as presented by Bacewicz for LiZnAs: (F-43m) a = 5.939 ̊, and for LiZnP (F-43m) a = 5.755 ̊ [24]. 


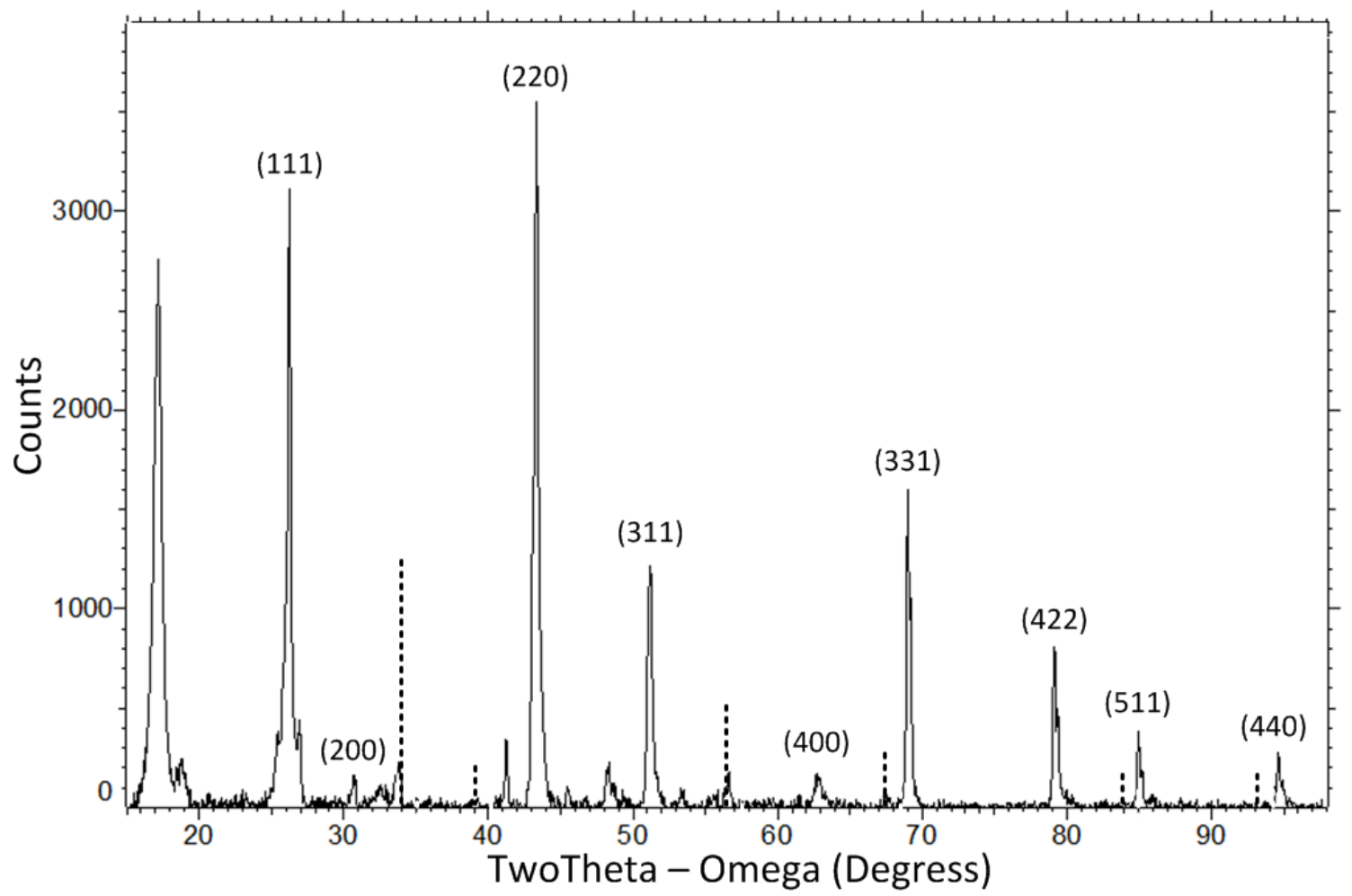

374

376

377

Fig. 6. LiZnAs phase identification scan collected on a Bruker AXS D8 ADVANCE equipped with Cu radiation. The dashed vertical lines are experimental data of cubic $\mathrm{LiO}$ [29].

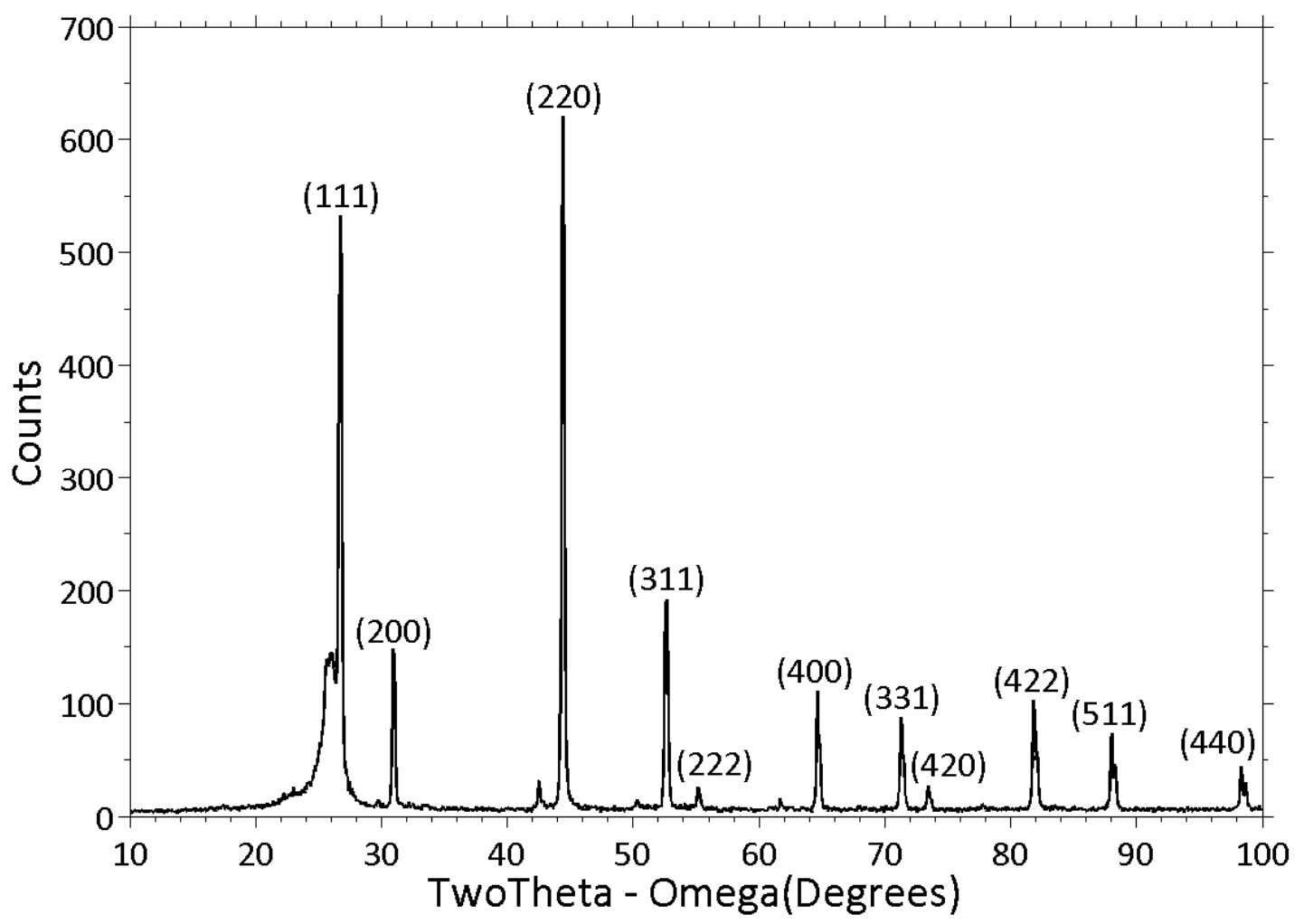

Fig. 7. LiZnP phase identification scan collected on a Bruker AXS D8 ADVANCE equipped with Cu radiation. 


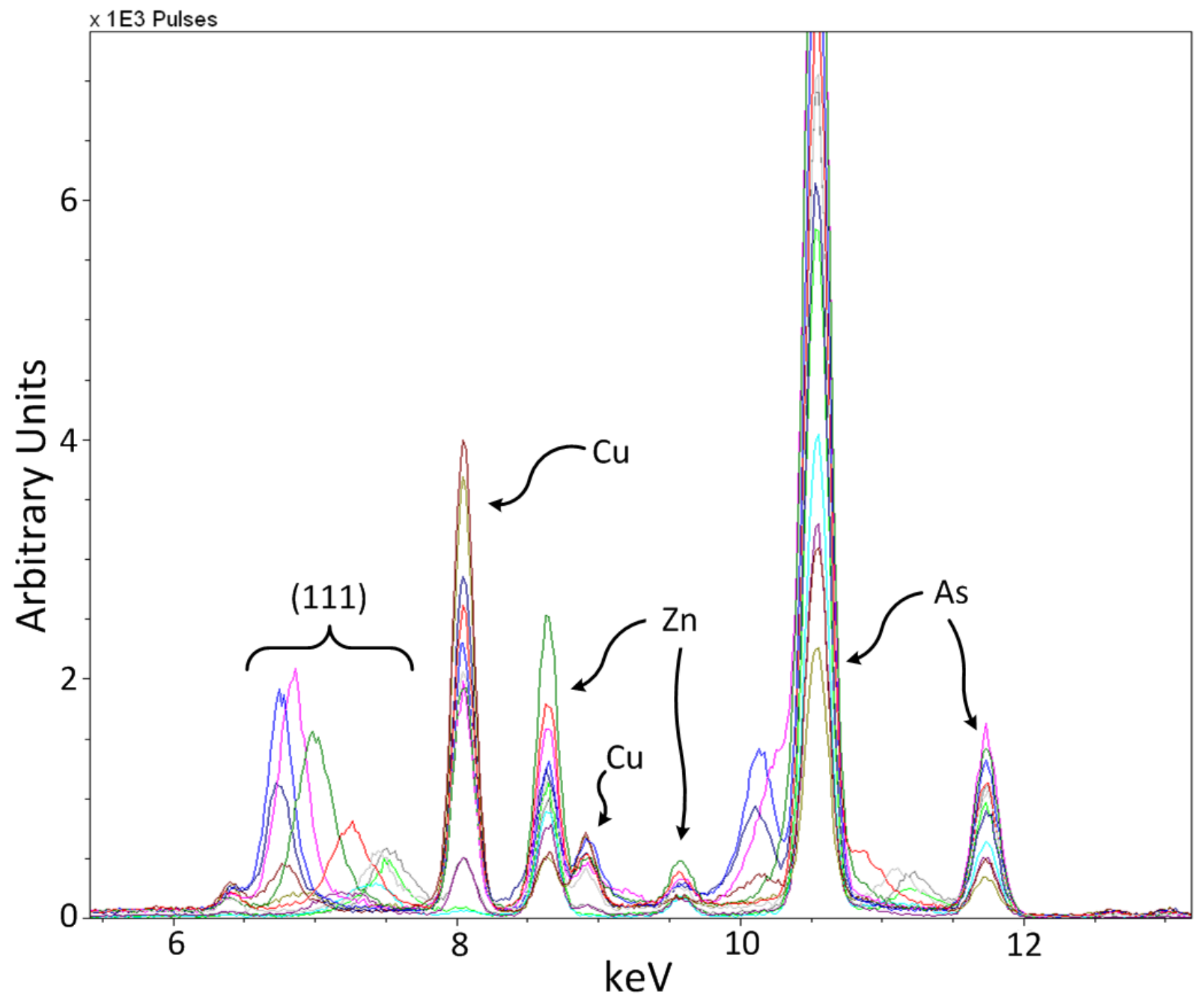

Fig. 8. Energy dispersive XRD scan of a single crystal sample of LiZnAs. Apparent iron, calcium and copper impurities were found in this specific sample as specified by the characteristic x-ray peaks. Other characteristic xray peaks for arsenic and zinc were located as labeled. Notice the Laue peaks of the (111) planes between 7.0 and $9.5 \mathrm{keV}$, which were used to verify the orientation of the sample. 


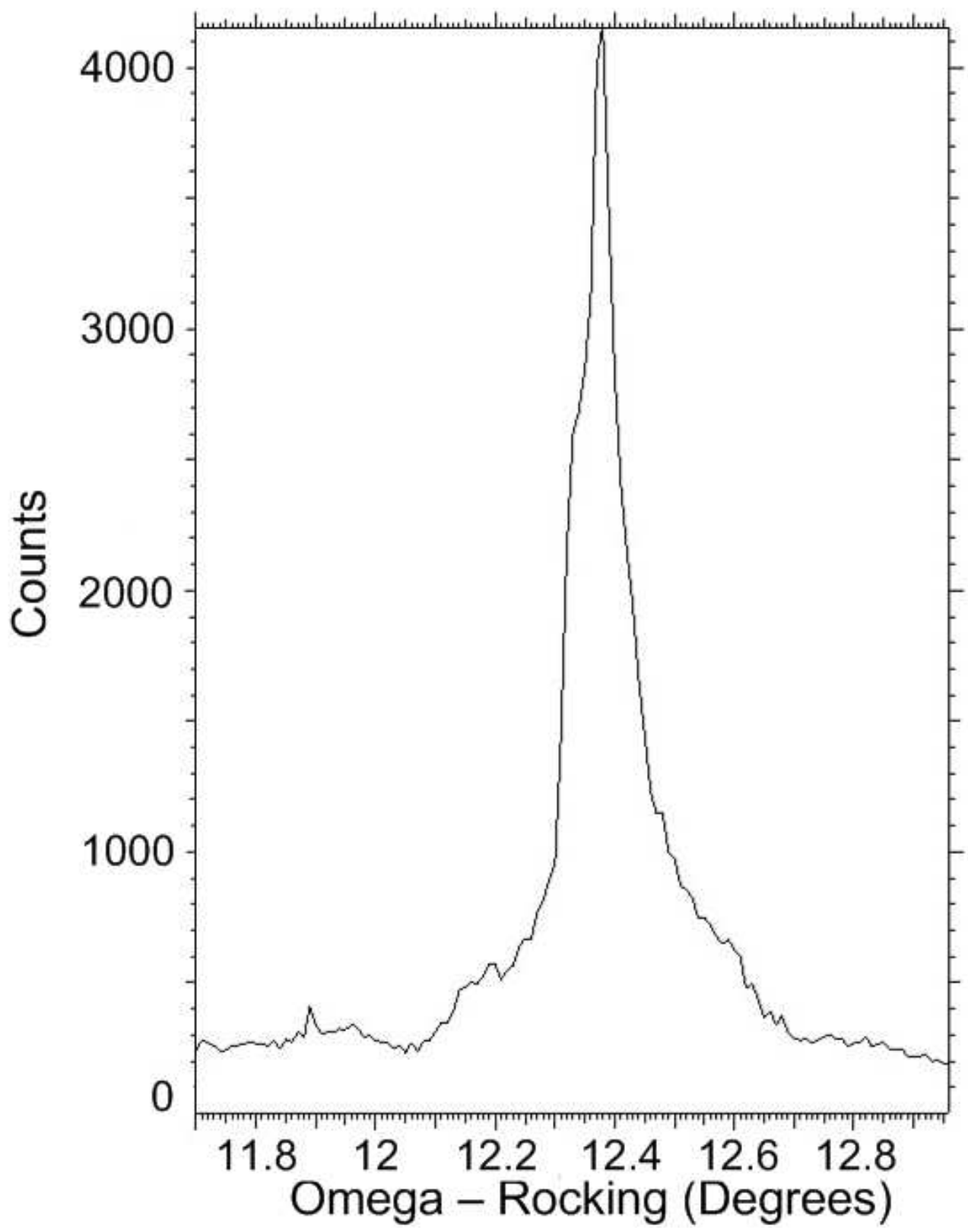

Fig. 9. High resolution rocking curve of the single crystal LiZnAs sample of the (111) family of planes where a 389 FWHM of $0.097^{\circ}\left(349.2\right.$ arc sec) was determined with a beam divergence of $0.004^{\circ}$.

390 


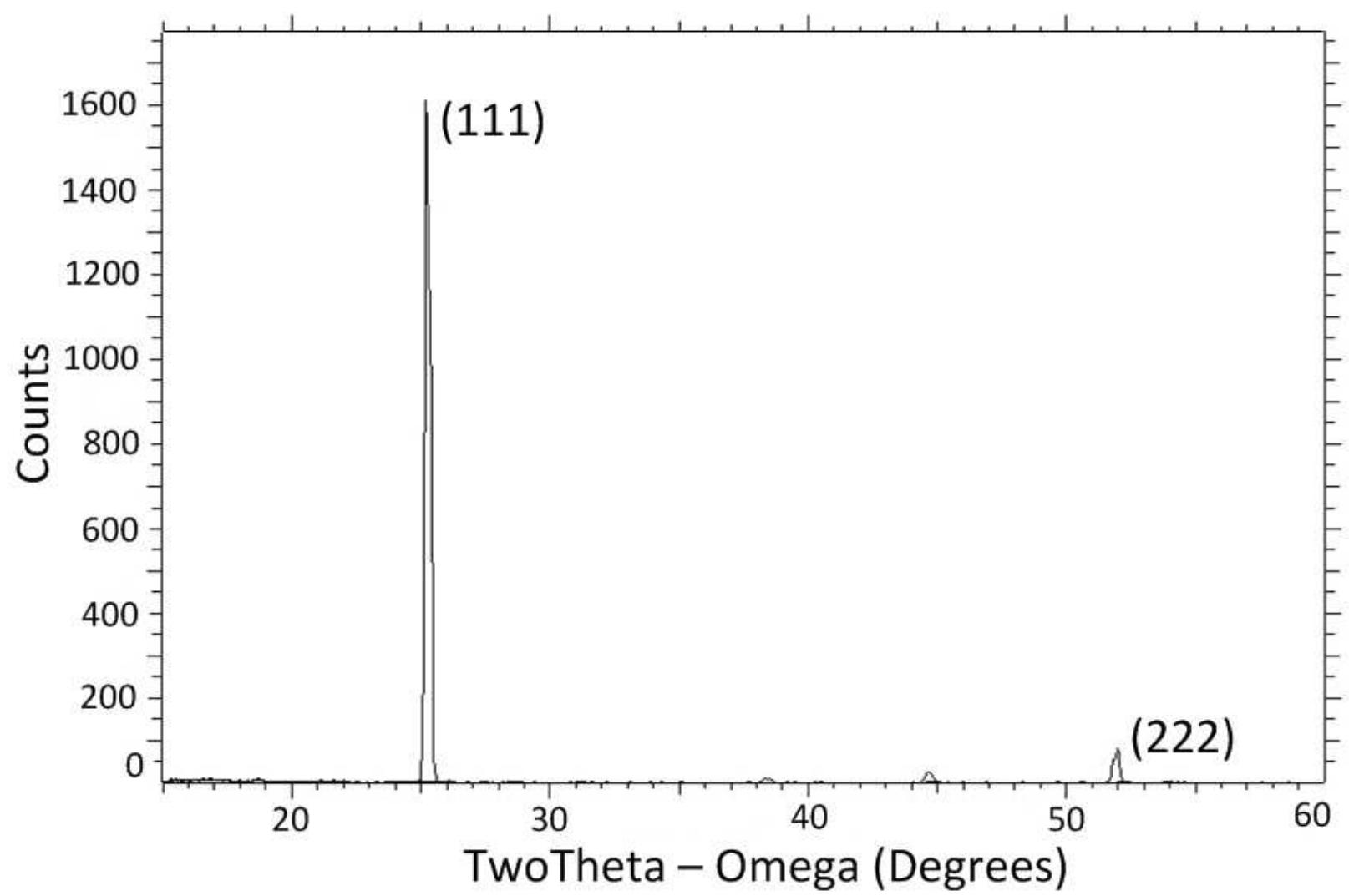

Fig. 10. Phase identification of the (111) family of planes in the single crystal LiZnAs sample.

Table 1. The ICP-OES results from Galbraith Laboratories Inc.

\begin{tabular}{ccccccccc}
\hline & \multicolumn{2}{c}{ Sample: LiZnP } & \multicolumn{3}{c}{ Sample: LiZnAs (1) } & \multicolumn{2}{c}{ Sample: LiZnAs (2) } \\
\hline Analysis & $\begin{array}{c}\text { Result } \\
(\%)\end{array}$ & $\begin{array}{c}\text { Molar } \\
\text { Concentration } \\
(\mathrm{mol} / \text { Vol) }\end{array}$ & Analysis & $\begin{array}{c}\text { Result } \\
(\%)\end{array}$ & $\begin{array}{c}\text { Molar } \\
\text { Concentration } \\
(\mathrm{mol} / \text { Vol) }\end{array}$ & Analysis & $\begin{array}{c}\text { Result } \\
(\%)\end{array}$ & $\begin{array}{c}\text { Molar } \\
\text { Concentration } \\
(\mathrm{mol} / \text { Vol) }\end{array}$ \\
\hline $\mathrm{P}$ & 31.3 & 0.010 & $\mathrm{As}$ & 41.3 & 0.0055 & As & 50.7 & 0.0068 \\
$\mathrm{Li}$ & 7.47 & 0.010 & $\mathrm{Li}$ & 4.83 & 0.0070 & $\mathrm{Li}$ & 5.01 & 0.0072 \\
$\mathrm{Zn}$ & 64.6 & 0.010 & $\mathrm{Zn}$ & 32.1 & 0.0049 & $\mathrm{Zn}$ & 41.5 & 0.0063 \\
\hline Total & 103.37 & & Total & 78.23 & & Total & 97.21 & \\
\hline
\end{tabular}

